\title{
SIMPOSIO INTERNACIONAL SOBRE SICOLOGIA Y EDUCACION
}

Durante los días comprendidos entre el 8 y el 12 de octubre del presente año, tuvo lugar en la ciudad de Bogotá, el Simposio Internacional sobre Sicología y Educación, el cual fue organizado por la Universidad Pedagógica Nacional a través de su Facultad de Educación.

El Comité organizador de tal evento estuvo conformado por los profesores Nora Pabón de Restrepo, Isabel de Chalem, Betty Monroy y Carlos Pereira.

El Simposio Internacional sobre Sicología y Educación tuvo como objetivos, los siguientes:

1. Precisar algunas relaciones teórico-metodológicas entre la Sicología y la Educación.

2. Analizar avances investigativos en el campo sicológico y los niveles de aplicación a la Educación.

3. Precisar criterios curriculares con respecto al contenido del área sicológica en la formación de profesionales de la Educación.

\section{Metodología:}

En lo referente al Simposio se trabajó con base en conferencias magistrales, sistemas de preguntas y mesas redondas. Para los talleres, además de las exposiciones, se utilizaron ampliamente técnicas audiovisuales y trabajo en grupo.

\section{Conferenciantes:}

EDGAR HEINEKEN, Delegado de la UNESCO en CLACSO y Profesor en varias universidades alemanas.

ARTHUR STAATS. Profesor e investigador de la Universidad de Hawai.

JAVIER AGUILAR. Profesor y Jefe del Departamento de Sicología Educativa en el postgrado de la UNAM.

FLORENTE LOPEZ. Profesor titular a nivel de postgrado en la UNAM.

BENJAMIN ALVAREZ. Director de la Facultad de estudios Interdisciplinarios de la Universidad Javeriana Bogotá.

HERNAN ESCOBEDO y JOSE MUÑOZ, Investigadores del Centro de Investigaciones de la Universidad Pedagógica Nacional.

MARIA EUGENIA COLMENARES, Sicóloga egresada de la Universidad de Ginebra.

FELIX BUSTOS. Sicólogo vinculado al Ministerio de Educación Nacional en la División de Diseño y Programación Curricular de Educación Formal. 
JAIME GONZALEZ. Sicólogo vinculado a la Universidad de Antioquia a través de docencia y programas de orientación y Consejería.

JAVIER DE NICOLO. Sacerdote Director del Programa Boscoma.

NEVA MILICIC, de la Universidad de Chile.

HENRY GRANADA. Profesor del Departamento de Sicología de la Universidad del Valle.

JOSE A. SANCHEZ. Decano de la facultad de Sicología de la Universidad Católica.

EDUARDO PONCE DE LEON y ROBERTO HERNANDEZ, profesores de la Universidad Católica.

Los conferenciantes disertaron sobre sus más recientes trabajos en el campo de la Sicología y su relación con los aspectos educativos, a nivel de educación especial, educación preescolar, educación primaria, Currículum, Consejería, Madurez escolar, Didáctica de la Sicología y los nuevos aportes de las diferentes tendencias sicológicas en lo referente a la educación.

\section{Talleres}

Durante los días 11 y 12 de octubre y con intensidad de ocho (8) horas cada uno, se llevaron a cabo siete (7) talleres de profundización en los temas tratados durante las conferencias. Dado el interés de los asistentes se repitieron algunos de estos talleres.

\section{Taller 1}

“Aportes de la Sicología Cognoscitiva a la Educación”, Edgar Heineken.

\section{Taller 3 y $3 A$}

"Composición de Lectura”, Javier Aguilar.

\section{Taller 4 y $4 \mathrm{~A}$}

“Aprendizaje de la Escritura y el Cálculo", Arthur Staats.

\section{Taller 5}

"Métodos de Campo para el estudio de la Conducta". Florente López.

\section{Taller 6}

“La educación del incapacitado mental”. Blanca Inés de Ballejo y Carmen de la Rosa.

\section{Taller 7}

“Madurez Escolar", Neva Milicic. 
Durante el Simposio también se discutieron algunos planteamientos curriculares para la formación de educadores, a través de una mesa redonda con algunos profesores invitados. 